\title{
Problem-Based Learning and Industrial Engineering
}

\section{Dr. Abhijit Gosavi, Missouri University of Science \& Technology}

Abhijit Gosavi obtained a Ph.D. in Industrial Engineering from the University of South Florida in 1999. He also has a B.S. and a master's degree in Mechanical Engineering (both degrees were from India). His research interests include simulation-based optimization, engineering education, Markov decision processes, revenue management, and productive maintenance. He has published in numerous journals in areas related to his research. He is currently an Assistant Professor in the Department of Engineering Management and Systems Engineering in Missouri University of Science and Technology, located in Rolla, MO.

\section{Dr. Jane M. Fraser, Colorado State University, Pueblo}

Jane M. Fraser is Chair of the Department of Engineering at Colorado State University, Pueblo. She was formerly on the faculty at the Ohio State University and Purdue University. She has a B.A. in mathematics from Swarthmore College and a M.S. and a Ph.D. in industrial engineering and operations research from the University of California, Berkeley. 


\title{
Problem-Based Learning and Industrial Engineering
}

\begin{abstract}
Problem-based learning (PBL), also called inductive learning, is a well-known approach for teaching engineering courses. We undertake a study of concepts that can be taught via PBL, along with an analysis of courses and topics in the industrial engineering (IE) curriculum suitable for PBL. While in the traditional deductive style of teaching, one usually starts with explanation of principles followed by examples, $\mathrm{PBL}$ is primarily characterized by providing examples first and then generalizing to the underlying principles. A great deal of literature cites evidence of PBL being more effective than deductive learning. However, PBL also provides numerous challenges to the instructor - especially to those used to teaching deductively. Some of these challenges include taking a fresh look at how the same topics that were taught via deductive methods can potentially be taught using PBL, the tradeoff between the increased amount of time it consumes with the amount of material to be covered in the class, and ensuring that the student is not confused and does not walk away with an incorrect understanding of the topic.
\end{abstract}

While PBL has been studied in the literature in the context of engineering in general, there is little by the way of how PBL can be used in IE courses. Hence, we first enumerate a number of topics from some IE courses that could potentially be taught with PBL. Then, we illustrate how PBL can be used in IE with two specific examples drawn from the following courses:

Probability and Statistics and Discrete-Event Simulation. Finally, we present a discussion on IE topics/concepts that are perhaps unsuitable for the PBL style.

\section{Introduction}

A great deal of evidence in the literature shows that engineering students in all disciplines learn better via generalizing from examples. This kind of learning is often called problem-based learning (Wilkerson and Gigselaers ${ }^{13}$; Grasha $^{4}$; Woods ${ }^{14}$ ), which is commonly abbreviated as PBL. In PBL, one begins with an example (or multiple examples), and then generalizes to the underlying principle. PBL is opposed to the deductive learning (teaching) style in which the principle is introduced first and examples follow (Felder and Silverman²). But PBL has many dimensions other than just being diametrically opposed to deductive learning: PBL is experiment-based (McKeachie ${ }^{7}$ ); PBL is based on inquiry (Hmelo-Silver et al. ${ }^{5}$ ); PBL uses openended examples/problems (Prince and Felder ${ }^{9}$ ). The evidence that PBL is very effective has appeared in numerous studies (that we will specify later).

The focus of this work is to present an analysis of how PBL can be used within the IE curriculum; we present examples from two important IE courses: (i) Probability and Statistics and (ii) Discrete-Event Simulation. We believe that there is no literature that analyzes the use of PBL within IE. Hence, this work seeks to fill this gap in the literature. We caution the reader that our work is based on a preliminary study of what should work and what is not likely to work in IE when PBL is used. Further, every instructor is unique and what may work for one instructor may not work for another. But should an instructor choose to use PBL in an IE course, we hope that our account here will be helpful in providing a roadmap for developing PBL-based teaching material. 
The rest of this paper is organized as follows. We first discuss the advantages of using PBL in general. Thereafter, we present a discussion on why deductive teaching seems to be a preferred approach in textbooks and the impact that has on our teaching in the IE discipline. Then, in a separate section, we cover the topics that can be potentially covered using PBL in IE, focusing on the two specific IE courses named above. In that section, we also present some examples of discussions that the instructor can have in the classroom in order to extract the greatest advantage of the PBL style. Finally, we conclude with a summary of our discussion and enumerate some topics that are perhaps best taught via a deductive mechanism.

\section{Advantages of PBL}

A few studies that show PBL to be beneficial are: Pierrakos et al. ${ }^{11}$, Mergendoller et al. ${ }^{8}$, Dochy et al. ${ }^{1}$, Patel et al. ${ }^{10}$, and Vernon and Blake ${ }^{12}$. Some studies, however, point in the opposite direction, e.g., Kirschner et al. ${ }^{6}$, but these have been far outnumbered by those that show positive outcomes with PBL. Some of the positive outcomes shown in the literature that are particularly relevant to us are:

a. Students retain what they have learned over a long period of time (Dochy et al. ${ }^{1}$ ).

b. Students can generalize what they have learned to other areas in related fields (Patel et $\mathrm{al}^{10}$ ).

c. Students are encouraged to be curious (Hmelo-Silver et al. ${ }^{5}$ ).

d. Students gain more domain knowledge (Mergendoller et al. ${ }^{8}$ ).

e. Students are encouraged to think simultaneously rather than sequentially and question prior learning $\left(\right.$ Gallow $\left.^{3}\right)$.

It is necessary to explain how these claimed benefits can result from using PBL. PBL forces students to think on their own. Very importantly PBL helps them recognize that many concepts in IE were developed for a reason and that they did not always exist in the body of knowledge. This thought process, especially when stimulated in the context of many fundamental concepts related to the subject (e.g., Discrete-event simulation, Production Planning and Control, Linear and Non-linear Programming, and Quality Control), is often the reason for outcomes $c$ and $e$. When students are forced to think and research a topic on their own, they have been seen to have an increased long-term memory of the topic (outcome $a$ ). Outcome $b$ can be an indirect consequence that is likely to occur when students start seeing the whys behind the fundamental topics. The outcome $d$ is seen when students spend time researching the topic on their own.

In contrast to PBL, deductive learning has some obvious drawbacks. One advantage with deductive learning is that there is no danger of confusing the student. However, it is not easy to engage students in discussion since you (as the instructor) are expected to provide the examples after you have discussed the principle, and this can make a mathematical subject appear dry. Also, unless the instructor goes back and provides some historical perspective, the student does not see how and why a given concept was invented or derived. Thus, it appears that in contrast, PBL makes for a more engaging style for communicating information, since students are likely to be more attentive (Hmelo-Silver et al. ${ }^{5}$ ).

The positive outcomes that can accrue from using PBL are accompanied by numerous challenges for the instructor. The most important challenge is the amount of time PBL requires in the 
classroom. It is not difficult to see where this increased amount of time is needed: it is in discussions on generalizing from the example that are not needed in a deductive style of teaching. These discussions are likely to result in numerous questions from students, and answering them is time consuming. Note that many of these questions, coming from students who are trying to grasp the topic, could be potentially misleading, and hence the instructor has to spend time in correcting the path of the discussion. The second challenge for the instructor is ensuring that the student does not walk away with an incorrect understanding of the concept. Thirdly, the instructor used to teaching deductively is likely to have his/her own learning curve in getting used to a PBL style. This could result from the fact that most textbooks do not use PBL, since deductive learning is likely to take a lot of space. It could also result from the fact that teaching a concept that the instructor learned deductively as a student may require creative thinking on the instructor's part. Hence, preparing material to be used in a PBL format is likely to increase the time needed by the instructor to prepare for class. Finally, we must add that a PBL-style discussion in the classroom is likely to require a one-on-one discussion with many, if not all, students, and increasing the classroom size beyond a reasonable number (e.g., twenty) is likely to provide a significant obstacle to using PBL in the classroom. It is most likely that in a class of size say eighty, students are unlikely to engage in the kind of discussions that PBL would require.

\section{Use of Deductive Learning in Textbooks}

Interestingly, most IE textbooks use a deductive approach. While we need not name such texts, it will be challenging to find a textbook on Probability, Statistics, Quality Control, Production Planning, Operations Research (stochastic or deterministic), or Discrete-Event Simulation that uses a PBL format. It is natural hence that the instructor follows a deductive format. However, with some effort, it is possible to develop the material needed for supplementary reading that can be used with texts (written in deductive style) to teach some concepts in a PBL style. We will provide some examples later.

Since engineers are required to solve problems rather than model phenomena (which is more typical of what is expected of social scientists and economists), it is usually vitally important that they develop the skills needed to apply these ideas in areas other than those in which they were introduced (see outcome $b$ above). This is an aspect that deductive learning stays away from, and as a result, IE textbooks, which the authors have found to be highly deductive, do not train students in the ability to generalize beyond the subject at hand. (This is probably true of other disciplines, but the authors do not have the experience/expertise to comment on that.) The ability to reason that PBL helps students acquire is likely to be of a significant advantage in seeing the big picture - a skill that the industrial engineer is supposed to possess. It appears thus that IE instructors interested in using a PBL style need to use supplementary material that goes with the textbook they are using.

\section{PBL-Friendly Concepts in IE Curriculum}

Our goal in this section is to highlight a subset of concepts in IE that can be taught using a PBL style. It is not our intent to provide an exhaustive list. Also, after providing a list, we then provide some guidelines on how to develop PBL material for introducing a concept that has been 
taught using a deductive style in textbooks.

Some of the core high-level courses taught in the curriculum of IE (or a closely related discipline) are (in no particular order):

- Engineering Economics

- Probability and Statistics

- Operations Research

- Production Planning and Control

- Facilities Layout

- Quality Control

- Discrete-Event Simulation

- Human Factors

- Safety and Ergonomics

In what follows, we first enumerate some of the main concepts in some of the above mentioned courses that can be taught in a PBL style.

Engineering Economics:

- Interest rate formulas

- Decision making using Net Present Value

Probability and Statistics:

- Random variables

- Means, variances, and standard deviations

- Addition and multiplication laws of probability

- Random variables

- Conditional probabilities

- Distribution and density functions

Operations Research:

- Decision variables

- Objective functions

- Optimal solution(s)

- Linear programs

- Integer programs

- Average wait in a queue

- Average Length of a queue

Production Planning and Control:

- Forecasting

- Economic Order Quantities and Newsboy Models

- Continuous and periodic review

- Inventory measurement

- Bullwhip effect

- Scheduling 
- Making to order Vs making to stock

- Push Vs Pull

Facilities Layout:

- Designing area requirements in a layout

- Product layouts Vs process layouts

- Cellular layouts

- Material handling equation for material handling cost

- Optimal layouts that minimize the material handling cost

- Designing optimal locations for facilities

\section{Quality Control:}

- Acceptance Sampling

- Tolerances

- Online process control

- Control charts

- Sampling

\section{Discrete-event Simulation:}

- The need for simulation models

- Random number generation

- Confidence intervals

- Distribution fitting

We now discuss two specific concepts that can taught in a PBL style rather than in the deductive format used in most books. We begin with the elementary concept of sample standard deviation when the population mean is unknown. In a deductive style, it is often taught as the following formula:

$$
\sigma=\sqrt{\frac{\sum_{i=1}^{n}\left(x_{i}-\bar{x}\right)^{2}}{n-1}}
$$

where $x_{i}$ denotes the $i$ th sample, $\bar{x}$ denotes the sample mean, and $n$ denotes the number of samples; the sample mean is defined as:

$$
\bar{x}=\frac{\sum_{i=1}^{n} x_{i}}{n} .
$$

The instructor then may (or may not!) explain why one uses (n-1) rather than $n$ in the above definition of sample standard deviation. The process described above is very commonly used in most textbooks to introduce the concept of standard deviation. We now discuss how this can be done in a PBL-friendly manner. Our discussion follows a conversational style typically adopted in teaching by the instructor. 
We provide you (students) the following sample:

$(1,4,3,5,7)$.

Let’s compute its arithmetic mean and denote it by $\bar{x}$. Then, clearly,

$$
\bar{x}=\frac{1+4+3+5+7}{5}=4
$$

It is not difficult then to generalize this to $\bar{x}=\frac{\sum_{i=1}^{n} x_{i}}{n}$ (although this generalization may not be necessary, since most students are familiar with arithmetic mean from high school). We are interested in generating an estimate of the "average value" of the deviations above from the mean. The goal is to determine how much variability from the mean exists in the sample.

One approach could be to measure the deviation of each sample from the mean and then average it. Let's see what happens if we try to measure it. The deviations of each of the samples from the mean, 4, are:

$$
\begin{gathered}
(1-4),(4-4),(3-4),(5-4),(7-4) \text { or } \\
(-3),(0),(-1),(1),(3)
\end{gathered}
$$

Let's compute the mean of these deviations:

$$
\frac{-3+0-1+1+3}{5}=0 \text {. }
$$

Of course, at this point, it is necessary to convince the students via other examples, or from the formula of the deviation, that the arithmetic mean of the deviations will always be 0 , regardless of the data. Clearly, then, developing an estimate of the average value of the deviation will not provide any useful information. At this point, we must look for an alternative metric that measures the "average value" of the deviations from the mean. Let's see what happens if we square the deviations and then add them. Will they sum up to zero too?

$$
(-3)^{2}+0^{2}+(-1)^{2}+(1)^{2}+(3)^{2}=18
$$

Clearly, they did not sum up to zero. Now, note that although we have 5 samples, we do not have 5 independent samples: if we are supplied with any (n-1) samples from the set of deviations above, we can predict the value of the $n$th sample. Thus, for instance, if we know, the first four samples, $-3,0,-1,1$, then since the samples sum to zero, clearly the last sample must be $-(-3+0-1+1)=3$. Of course, this must be shown to be true for any sample, not just the last sample. Once the student sees this, it is not hard to convince the class that we have in fact only $4(=n-1)$ independent samples here and not $5(=n)$. Hence, if we now average the five squared deviations, we should divide the sum by 4 and not 5 . Thus, a reasonable metric for the "average" value of the squared deviation will be: 


$$
\frac{(-3)^{2}+0^{2}+(-1)^{2}+(1)^{2}+(3)^{2}}{4} .
$$

To obtain the "average" value of the deviation in the same units as the sample, it appears intuitive to take the square root of the above:

$$
\sqrt{\frac{(-3)^{2}+0^{2}+(-1)^{2}+(1)^{2}+(3)^{2}}{4}} .
$$

One can now explain that what has been obtained above is in fact the so-called "sample standard deviation" and is a reasonable measure of an "average" value of the deviation from the mean. We can then also show that it generalizes to the formula of the standard deviation.

The above PBL-style approach to teaching sample standard deviation has many advantages. First of all, students recognize the need for dividing by $(n-1)$ rather than by $n$. Secondly, they see the standard deviation as a metric that is indeed useful since it is some sort of an estimate of the "average" value of the deviation. Thirdly, they recognize that statisticians have indeed done something remarkably clever here in defining the sample standard deviation, since a naively computed arithmetic mean of the deviations is always zero, regardless of what values the samples have. Of course, as alluded to above, this discussion may take up to 15-20 minutes, whereas a deductive approach may require less than 5 minutes of class time.

We now consider another example; this example is chosen from discrete-event simulation. Here our goal is to show that PBL teaching may not require the elaborate "reverse engineering" of a concept that was needed in the example above (standard deviation). Here the PBL approach will be simply to illustrate the use of a concept before formally defining it. The example we consider is the concept of computing confidence intervals on means of random variables (that are outputs in a simulation model) via the replication method, which is an important topic typically covered in the simulation course. Here, we will restrict our discussion to motivating the need for generating confidence intervals (rather than the actual formula and how it is derived).

If possible, we run a simulation model repeatedly, using a new set of random numbers, to obtain values for means of output random variables in our simulation model. The standard practice is to use values obtained from each replication as samples to compute the mean and the standard deviation in the sample. The standard deviation, under suitable assumptions, is then used to compute the confidence interval on the mean. Usually, when the student performs the associated arithmetic, it is assumed that this is a necessary step that accompanies the computation of the mean of the random variable we are interested in. It is also explained in many texts that as one increases the number of replications (or samples), the estimate of the mean becomes more accurate. A PBL-friendly explanation of this topic would be slightly different.

When one obtains the mean of a random variable from a theoretical closed form (mathematical) model, one obtains the exact value of the mean (provided the mathematical model is exact.) However, when one runs simulation models, the mean we obtain from running the simulation is 
only an estimate and not its exact value (provided it is a stochastic system). The instructor should run several replications, without initializing statistics between the replications, and then show the differing estimates of the mean for the same random variable produced via the different replications.

At this point, the instructor should write out the means obtained from the different replications on the board, and then explain that when standard deviations are to be estimated from samples, the samples need to be independent. When the simulation is run with a new set of random numbers (the meaning of a replication in the context of computer simulation), independent estimates of the random variable's mean are in fact generated. Now, when the standard deviation is computed on the board from the samples obtained, the confidence interval on the mean should be computed using the $t$-statistic.

Now, some additional replications should be run to generate additional values for the random variable whose mean we are interested in. First, the standard deviation and mean should be recalculated. Thereafter, the confidence interval should be recalculated. It is then necessary to show that when the additional values from the replications are used, the confidence interval shrinks in its width. At this point, the instructor can explain that the confidence interval computed in this style is in fact the error in estimating the mean - that gradually diminishes as one performs additional replications.

\section{Conclusions}

Our goal in this paper was to highlight the advantages of using PBL in the IE curriculum. We cited some of the well-known advantages of PBL for teaching engineering, and then discussed how they also apply to the IE discipline. We also discussed the challenges posed by PBL. Thereafter, we enumerated some concepts that can be taught in a PBL-friendly manner in a host of courses taught within an IE curriculum. We concluded with an account on two specific topics that can be taught in a PBL style although they are invariably covered in a deductive style in most books. We now present a list of topics that perhaps ought to be covered in a deductive style.

Discrete-event simulation: Event clocks; model verification and validation.

Probability and Statistics: joint probability density functions and joint distribution functions; Chi-squared test.

Facilities Layout: Cell formation via similarity coefficients; warehouse designing.

These topics, we believe, are more appropriately taught in a deductive style because the logic behind them is more difficult to extract from students, because either an in-class problem cannot substitute for the experience that led to the development of the idea, or a PBL approach would take excessive amounts of time. It is also perhaps true that using a PBL style for more complicated/advanced topics may not be feasible, since the instructor may not be aware of the entire sequence of the historical development of the process. For instance, expecting students to see on their own (in a brainstorming session) how cells can be formed via similatiry coefficients 
may not be appropriate. Similarly, it is unreasonable to expect the students to derive, on their own, the basic ideas underlying the construction of a simulation clock.

Clearly, a lot of further research can be performed in this area. A more exhaustive study needs to be carried out that should cover many of the concepts identified above; further it is also important to determine which of the findings that are claimed to hold for engineering also apply to IE. It will be also interesting to (statistically) test the differences in outcomes from a section of an IE class that uses deductive teaching versus another section that uses PBL teaching.

\section{References}

1. Dochy, F. M. Segers, P. Van den Bossche. Effects of problem-based learning: A meta-analysis. Learning and Instruction, 13, 533-568, 2003.

2. Felder, Richard and Linda K. Silverman. Learning and Teaching Styles in Engineering Education, Engineering Education, 78(7), 674-681, 1988.

3. Gallow, D. What is problem-based learning? http://www.pbl.uci.edu/whatispbl.html, 2011.

4. Grasha A. F. An integrated model of teaching and learning style: In Teaching with Style: A Practical Guide to Enhancing Learning by Understanding Teaching and Learning Styles. San Bernardino, Calif: Alliance Publishers, 1996.

5. Hmelo-Silver, C.E., Ravit Golan Duncan, and Clark A. Chinn. Scaffolding and achievement in problembased and inquiry learning: A response to Kirschner, Sweller, and Clark (2006), Educational Pyschologist, 42(2), 99-107, 2007.

6. Kirschner, P. A., J. Sweller and R.E. Clark. Why minimal guidance during instruction does not work: An analysis of the failure of constructivist, discovery, problem-based, experiential, and inquiry-based teaching. Educational Psychologist, 41, 75-86, 2006.

7. McKeachie, W.J. Teaching Tips, $10^{\text {th }}$ edition, Houghton Mifflin, NY, 1999.

8. Mergendoller, J. R., N.L. Maxwell and Y. Bellisimo. The effectiveness of problem-based instruction: A comparative study of instructional method and student characteristics. Interdisciplinary Journal of Problem based Learning. 1, 49-69, 2006.

9. Prince, $\mathrm{M}$ and R.M. Felder. Inductive Teaching and Learning Methods: Definitions, Comparisons, and Research Bases. Journal of Engineering Education, 95(2), 123-138, 2006.

10. Patel,V. L., G.J. Groen,G. and G.R. Norman. Reasoning and instruction in medical curricula. Cognition and Instruction, 10, 335-378, 1993.

11. Pierrakos O., R. Kander, E. Pappas, and R. Prins. An Innovative Engineering Curriculum at James Madison University: Transcending Disciplinary Boundaries Through Innovative Problem Based Learning Practices. ASME International Mechanical Engineering Congress \& Exposition, Boston, MA, 2008.

12. Vernon, D. T., and R.L. Blake. Does problem-based learning work? A meta-analysis of evaluative research. Academic Medicine, 68, 550-563, 1993.

13. Wilkerson, L. and W.H. Gigselaers (eds.). Bringing problem-based learning to higher education: Theory and Practice, in New Direction for Teaching and Learning, No 68, San Franscico: Jossey-Bass, 1996.

14. Woods, D. Preparing for PBL, third edition, McMaster University, online book http://chemeng.mcmaster.ca/pbl/pblbook.pdf, 2006. 